\title{
Wound repair during arm regeneration in the red starfish Echinaster sepositus
}

Yousra BEN KHADRA ${ }^{2}$, Cinzia FERRARIO $^{\mathbf{1}}$, Cristiano DI BENEDETTO ${ }^{\mathbf{1 , 3}}$, Khaled SAID $^{2}$, Francesco BONASORO ${ }^{1}$, M. Daniela CANDIA CARNEVALI ${ }^{1}$ and Michela SUGNI ${ }^{1}$

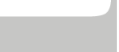

${ }^{1}$ Dipartimento di Bioscienze, Università degli Studi di Milano, 20133 Milano, Italy. ${ }^{2}$ Laboratoire de Recherche, Génétique, Biodiversité et Valorisation des Bioressources, Institut Supérieur de Biotechnologie de Monastir, Université de Monastir, Monastir, Tunisia.

${ }^{3}$ King Abdullah University of Science and Technology (KAUST), Biological and Environmental Sciences and Engineering Division, 23955-6900, Thuwal, Saudi Arabia.

*Corresponding author: Cinzia FERRARIO

Dipartimento di Bioscienze, Università degli Studi di Milano

Via Celoria 26, 20133 Milano, Italy

Phone: +390250314799 /Fax: +390250314781

e-mail: cinzia89.ferrario@alice.it

Running title: Starfish arm regeneration

Keywords: wound healing, subepidermal oedematous area, repair phase, arm regeneration, Echinaster sepositus, morphallaxis

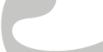

This article has been accepted for publication and undergone full peer review but has not been through the copyediting, typesetting, pagination and proofreading process which may lead to differences between this version and the Version of Record. Please cite this article as an 'Accepted Article', doi: 10.1002/wrr.12333 


\section{Abstract}

Starfish can regenerate entire arms following their loss by both autotomic and traumatic amputation. Although the overall regenerative process has been studied several times in different asteroid species, there is still a considerable gap of knowledge as far as the detailed aspects of the repair phase at tissue and cellular level are concerned, particularly in posttraumatic regeneration. The present work is focused on the arm regeneration model in the Mediterranean red starfish Echinaster sepositus; in order to describe the early cellular mechanisms of arm regeneration following traumatic amputation, different microscopy techniques were employed. In E. sepositus, the repair phase was characterized by prompt wound healing by a syncytial network of phagocytes and re-epithelialisation followed by a localized subepidermal oedematous area formation. Scattered and apparently undifferentiated cells, intermixed with numerous phagocytes, were frequently found in the wound area during these first stages of regeneration and extensive dedifferentiation phenomena were seen at the level of the stump, particularly in the muscle bundles. A true localized blastema did not form. Our results confirm that regeneration in asteroids mainly relies on morphallactic processes, consisting in extensive rearrangement of the existing tissues which contribute to the new tissues through cell dedifferentiation, re-differentiation and/or migration.

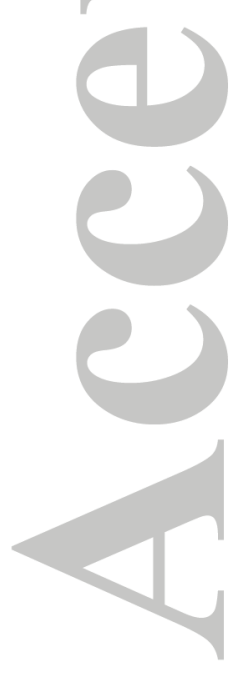




\section{Introduction}

According to Goss (1) and Mattson (2) regeneration is defined as the recovery process of lost or damaged tissues or organs due to injury. The regenerative capabilities vary a lot among Phyla depending on their adaptive value in terms of evolutionary advantage (3), and are expressed to a different extent in the diverse animal groups, independently of the phylogeny. The wide spectrum of regeneration processes in the diverse Phyla is evidence for the high adaptive value of this phenomenon. Although some vertebrates can display regeneration phenomena, i.e. reptile regenerate tails, amphibians lenses (4) or limbs (5) and mammals digits (6), these processes do not appear to be comparable to the amazing capacity of several invertebrates to repair and regrow extensive body parts and organs. However, the highest potential of invertebrate regeneration is not related to primitive conditions and is not only found in the lower metazoans, such as Hydra and planarians (7); many past and recent studies clearly demonstrated that extensive regeneration capabilities are displayed by highly evolved and complex animal groups, such as echinoderms and ascidians, both deuterostomes, which are excellent and promising experimental models for studies of regeneration $(8 ; 9)$. Indeed, echinoderms are known to have the greatest capacity for regeneration among deuterostomes and the established gene conservation between echinoderms and vertebrates (the sea urchin genome, recently sequenced, shows $70 \%$ homologies with the human genome (10)) suggests that findings from these animal models might be relevant to research addressed to mammalian regeneration.

Amongst echinoderms, asteroids are well known to be able to undergo extensive regeneration of arms following their self-induced amputation (autotomy) or traumatic loss/damage due to predation, accidents, etc. (8). The histological and cellular aspects of the regenerative process have been described in detail in a few works using Asterias rubens, Coscinasterias muricata and Leptasterias hexactis as model species $(11 ; 12 ; 13)$. According to these studies the overall 
regenerative process could be subdivided into three main phases: a repair phase, characterized by wound-healing processes; an early regenerative phase, characterized by first differentiation phenomena; and an advanced regenerative phase, characterized by proper arm regrowth. Depending on the presence or absence of a localized blastema of undifferentiated and proliferating cells after wound-healing, the regenerative events could be interpreted partly as epimorphic, partly as morphallactic processes, the borderline between these being apparently evident at macroscopical level, but rather ambiguous and still not well defined at cellular level. Indeed, in echinoderm regeneration the different mechanisms co-exist and this ambiguity is always present (8). Epimorphic processes with blastema formation are usually found in those situations where regeneration is a widely predicted event implying a standardized sequence of developmental phases following auto-mutilations: this is the case of post-autotomic regeneration of arms in crinoids (14) and some ophiuroids (15). In contrast, morphallactic regeneration seems to be a more complicated and slower process following accidental events whose extent is variable. This can be seen in post-traumatic arm-tip regeneration in asteroids and, more generally in all echinoderms, in all type of post-traumatic regeneration related to stress conditions (extreme mutilations, exposure to environmental pollution, etc.; $8 ; 16)$. In all these cases, the mutilation impact can vary greatly and the lost tissues cannot be restored by following a standardized developmental model, but they are regrown starting from the rearrangement of the old structures of the stump (17). With regard to the regenerative process of arm-tip in starfish, this is therefore considered mainly morphallactic: nevertheless, in terms of the cells that are involved, it can involve either pluripotent progenitor cells such as coelomocytes (18) or/and differentiated cells, which may undergo dedifferentiation or transdifferentiation (8).

Coelomocytes were found to be among the most actively involved elements during the repair phase of asteroid arm regeneration. In Asterias rubens, the aggregation of coelomocytes from 
adjacent tissues has been shown to significantly contribute to wound healing by clotting phenomena. The grouping of this non-proliferating cell type beneath the wound epidermis was considered also as the first line of defence following amputation (19). These cells undergo phagocytosis of cell debris and of microorganisms penetrating the internal body fluids from the wound area (11).

Although several previous studies have been focused on the early processes of arm regeneration in starfish, a comprehensive interpretation of the overall process is still missing and the cellular mechanisms and the origin of the involved cells are not yet well defined. For this reason further studies are needed: moreover, the characteristic diversity existing among species (in terms of morphology, physiology, ecology, and susceptibility to autotomy/traumatic amputation) makes each model very different from the others and therefore a detailed comparative analysis of different models appears to be very appropriate.

In this study the common Mediterranean red starfish Echinaster sepositus has been selected as a suitable experimental model. The reasons for this choice are the following. First, regenerating specimens of E. sepositus are not frequently found in the wild, indeed, this species neither autotomizes easily (personal observations) nor is subject to a high predation pressure; rather, it has maximized other defence strategies, such as the presence of subepidermal mucous glands containing toxic compounds (20). Therefore, we assumed that a "forced and unpredicted regenerative process" induced in a species with a low regeneration incidence might differ from the regeneration response of species frequently undergoing traumatic amputations by predation or self-induced autotomies such as $A$. rubens. Second, $E$. sepositus has been recently used as model species to investigate molecular aspects (homeobox genes) of arm regeneration (21) which need to be properly complemented by morphological investigations addressed to a detailed characterization of the tissue and cellular pattern. Consequently, using different microscopy techniques (light and electron) we intend to provide 
basic essential information on the main anatomical and ultrastructural aspects of the regeneration process in this species. The present paper, besides facilitating any future research efforts in this field, will provide the basic knowledge of the main cellular mechanisms during the early stages of regeneration which are so critical for guaranteeing the correct fulfilment of the subsequent phenomena of growth, morphogenesis and differentiation.

\section{Materials and methods}

\section{Ethics Statement}

All animal manipulations were performed according to the Italian law, i.e. no specific permits were required for the described studies since starfish are invertebrates. Echinaster sepositus is not an endangered or protected species. All efforts were made to minimize the animal suffering during experimental procedures. The specimens were released into their natural environment once the experimental procedures were completed.

\section{Animal sampling and regeneration tests}

Adult (diameter $\sim 12 \mathrm{~cm}$ ) specimens of Echinaster sepositus were collected by scuba divers at depth of 5-8 $\mathrm{m}$ from the Marine Protected Area of Portofino (Paraggi, Ligurian Sea, Italy) between November 2012 and April 2013. They were left to acclimatize for two weeks and maintained at $18^{\circ} \mathrm{C}$ in aerated aquaria filled with artificial sea-water (Instant Ocean, $37 \%$ ) for the whole experimental period. Chemical-physical sea water parameters were checked daily (temperature and salinity) or weekly (concentrations of nitrites, nitrates, $\mathrm{Ca}, \mathrm{Mg}, \mathrm{PO}_{4}$ and $\mathrm{pH}$ ) and promptly adjusted if necessary. Specimens were fed with small pieces of cuttlefish twice a week. Traumatic amputation of the distal third of one arm for each specimen was performed by scalpel. Animals were then left to regenerate in the aquaria for predetermined periods. The regeneration pattern was monitored at 1, 24 and 72 hour(s) post amputation (p.a.) and was also compared with that of normal non-regenerating arms. Four-six 
samples/individuals were analyzed for each stage. Regenerating arm tissues were removed including about $1 \mathrm{~cm}$ of the stump and were subsequently processed for the different microscopic analyses.

\section{Microscopic analyses}

Non-regenerating and regenerating tissues collected at different time points were analyzed by different microscopy techniques (light and electron, see below). Samples were initially observed and photographed under a LEICA MZ75 stereomicroscope provided with a Leica EC3 Camera and Leica Application Suite LAS EZ Software (Version 1.8.0).

\section{$\underline{\text { Light microscopy }(L M)}$}

Both thick (paraffin) and semi-thin (resin) sections were prepared. Briefly, for thick sections three samples per stage were fixed in Bouin's fluid for about one month to allow decalcification, washed in tap water, dehydrated in an increasing ethanol series, cleared with xylene, washed in xylene:paraffin wax solution $(1: 1)$ and embedded in paraffin wax $\left(56^{\circ}-58^{\circ}\right.$ C). Sagittal (longitudinal-vertical) sections $(5-7 \mu \mathrm{m})$ were cut and stained according to Milligan's trichrome technique. For resin sections, three samples per stage were fixed in SPAFG fixative (3\% glutaraldehyde, $1 \%$ para-formaldehyde, $7.5 \%$ picric acid saturated solution, $0.45 \mathrm{M}$ sucrose, $70 \mathrm{mM}$ cacodylate buffer) for one month to allow decalcification, washed in $0.15 \mathrm{M}$ cacodylate buffer and post-fixed in $1 \%$ osmium tetroxide in the same buffer for $2 \mathrm{~h}$. Samples were rapidly washed in distilled water and then in $1 \%$ uranyl acetate in $25 \%$ ethanol (2 hours), dehydrated in an ethanol series, cleared in propylene oxide, washed in propylene oxide:Epon 812-Araldite solution (3:1 for $1 \mathrm{~h}, 1: 1$ for $1 \mathrm{~h}, 1: 3$ for $1 \mathrm{~h}$ and $100 \%$ resin overnight) and embedded in Epon 812-Araldite. Samples were longitudinally sectioned using a Reichert Ultracut E with glass knives. The semi-thin $(1 \mu \mathrm{m})$ sections were stained with crystal violet and basic fuchsin. Thick and semi-thin sections were observed under a 
Jenaval light microscope provided with a DeltaPix Invenio 3S 3M CMOS Camera and DeltaPix Viewer LE Software.

\section{Scanning electron microscopy (SEM)}

After sagittal sectioning, the remaining paraffin embedded half-samples were also used for SEM analyses. Samples were washed several times with xylene for five days in order to completely remove the paraffin wax. Then they were washed in absolute ethanol and subsequently in HMDS and ethanol (in the proportions: 1:3, 1:1, 3:1) for 15 minutes each wash, and then washed 3 times in 100\% HMDS for 15 minutes. Finally all the processed samples were mounted on stubs, covered by a thin layer of pure gold (Sputter Coater Nanotech) and observed under a scanning electron microscope (LEO-1430).

\section{Transmission electron microscopy (TEM)}

For TEM analyses the same samples employed for semi-thin sections were cut with glass knives using the same Reichert Ultracut E. The obtained thin sections $(0.07-0.1 \mu \mathrm{m})$ were collected on copper grids, stained with uranyl acetate followed by lead citrate and finally carbon coated with an EMITECH K400X Carbon Coater. The thin sections were observed and photographed using a Jeol 100SX transmission electron microscope.

\section{Results}

Non-amputated arm: gross morphology and ultrastructure

A histological cross section of an intact arm, showing the main anatomical features, is illustrated in Figure 1A (for a review of asteroid anatomy see 20). The arm body wall hosts a series of calcitic ossicles which are organized in the typical three-dimensional structure, the stereom, whose "cavities" are filled by cells and ECM (Fig. 1B, for a review see 22). The ossicles are sandwiched between an inner and an outer dermis, formed by dense (DCT) and 
loose (LCT) connective tissue respectively. As reported in other starfish (23), most of the collagen fibres are arranged according to an orthogonal pattern. The body wall is internally completed by a coelomic myoepithelium (CE). In line with the literature (24), this consists of apical peritoneocytes and basal longitudinal myocytes and nerve processes (Fig. 1C). Additionally, bands of transverse myoepithelial cells formed rings of circular muscles, regularly distributed in the underlying connective tissue (see Fig. 4C). A regular series of prominent papulae juts out from the external arm surface. Numerous large mucous glands, typical of some Echinasteridae (20), are mainly present in the aboral dermis.

In the dermis several cells are distributed between collagen fibres. Besides the typical connective tissue cells (Fig. 2A), in our experimental model two other cytotypes could be frequently observed: myoepithelial cells and granulocytes (Fig. 2). Most often these two cell types were associated and enclosed together by a fibrous basal lamina, often in direct contact with collagen fibril bundles (Fig. 2A, C). Myoepithelial cells were scattered in the different dermis layers presumably organized in a loose network. They were characterized by a prominent euchromatic nucleus and their elongated cell processes were occupied mainly by bundles of myofilaments (Fig. 2B). The granulocytes were characterized by the presence of many circular and homogeneously electron-dense granules, approximately $1 \mu \mathrm{m}$ in diameter (Fig. 2B, C). Their cell processes often formed a sort of "chamber" where microvilli and cilia could be observed (Fig. 2C, D). These morphological features resembled those of secretory cells observed in the CE (24). Additionally, neurosecretory-like cells containing small electron-dense and roundish/oval granules (approximately $300 \mathrm{~nm}$ in diameter) were occasionally present in association with the previously described cytotypes; their morphological features were similar to those of the juxtaligamental cells (JLCs) or neurosecretory cells (24) described in the literature, which are considered to be distinctive elements of echinoderm mutable collagenous tissues (MCTs; 24). 
The main visible nervous component of the arm, the radial nerve cord (RNC), runs along the entire length of the ambulacral groove between the two rows of tube feet and ends into the optic cushion, the typical starfish photoreceptor structure. As in all asteroids, the RNC is typically V-shaped and continuous, in its lateral sides, with the epidermis (Fig. 1A, 3A). Indeed, the main nervous component of the RNC is the ectoneural system, showing the classic structure of a neuroepithelial plexus (26): this is separated from the adjacent hyponeural sinus by a thin connective tissue layer and a coelothelium (Fig. 3A). On both sides (mainly in its apical part) the V-structure encloses layered aggregates of presumptive hyponeural somata. In sagittal sections the ectoneural epithelium of E. sepositus appeared to be composed of three distinct layers: (i) a thin hyaline layer covering the surface of the cells and enclosing their cilia (Fig. 3B), (ii) a somatic zone (Fig. 3A), containing the nucleated portions of the epithelial supporting cells and neurons and (iii) a fibrillar zone (neuropile), made up of neurofibrillae intermixed with the axial parts of the supporting cells, containing intermediate filaments bundles (Fig. 3C).

\section{Regeneration}

\section{1 h p.a.: wound sealing}

As shown in whole mounts and in histological sections, immediately after removal of the armtip, the distal portion of the stump (about $3 \mathrm{~mm}$ ) strongly constricted (like a "haemostatic ring") thus sealing off the open ends of the perivisceral coelom (Fig. 4A, B). Papulae present in the contracted stump body wall appeared deflated, as a consequence of the reduced coelomic hydrostatic pressure. In the contracted area the collagen fibres of the DCT apparently became more densely packed and the coelothelium showed an extensively folded profile; the rings of circular muscle fibres underlying the coelothelium appeared to be

remarkably contracted, showing a more roundish and enlarged overall cross section when compared with those of more proximal area of the stump (Fig. 4C, D). This strong contraction 
pulled the first pair of tube feet towards the center of the wound. The injury appeared to be sealed also by the active contribution of coelomocytes which migrated through the coelomic fluid and formed clots closing the wound (Fig. 4E). Different cytotypes could be distinguished in this area, including both apparently undifferentiated cells and differentiated coelomocytes. The presumptive undifferentiated cells were small elements, roundish or oval in shape, with a very large nucleus and a scarce granular cytoplasm not containing any specialized organelles (e.g. phagosomes). Their surface did not show filopodial processes (Fig. 4F). The differentiated coelomocytes included different populations of migratory cells, recognizable by specific morphological features (shape, size, cytoplasmic inclusions, filopodial processes, etc.). The characterization of these cells is given in detail in stage 72 hours p.a. In any case, the microscopic analyses did not reveal any noticeable changes in the specific cellular composition of the $\mathrm{CE}$ either close to or far from the wound: no direct release of coelomocytes from the CE could be observed.

Even at this early stage the injured muscles showed first signs of reorganization and dedifferentiation phenomena and evidence of release of cells towards the wound area (Fig. $4 G)$.

\section{4 h p.a.: wound healing}

At $24 \mathrm{~h}$ p.a. the strong body wall contraction still persisted (Fig. 5A): the aboral body wall converged and folded towards the oral side (Fig. 5B). Within the end of the first day post amputation wound healing was almost completed, including the formation of a new thin epithelium (Fig. 5C, D). The epidermis, which seemed to converge centripetally from the edges towards the wound centre, provided cells for the formation of a new thin epithelial monolayer (Fig. 5E). At this stage, this latter was apparently composed only by partially dedifferentiated epidermal cells, derived from the stump, which were stretched in order to 
heal the injured area. These cells were initially flat and squamous with a prominent nucleus and cytoplasmic inclusions (granules, vacuoles, etc...) characteristic of the epidermal cells. Typical cell junction complexes, including both adherens and septate types, were detectable. Small microvilli immersed in a thin cuticle layer could be seen on the outer epithelial side, although a clearly recognizable basal lamina was still lacking (Fig. 5F). Numerous presumptive neural processes could be observed on the inner epithelial side (Fig. 5E). In some cases, beneath this wound epidermis a loose syncytial network of phagocytes was found containing several cytoplasmic inclusions and/or electron-transparent vacuoles, and creating wide and irregular lacunar areas. The syncytium appeared to form a barrier isolating extracellular matrix elements (e.g. collagen fibrils) and sparse cells that remained after ablation and/or had recently migrated to the wound area (Fig. 5G).

At this early stage the RNC was also healed: the regenerating fibrillar zone showed a rearranged pattern with a still disorganized architecture and in the most distal part only the cell body layer is visible (Fig. 5D).

\section{2 h p.a.: oedematous area formation}

The aboral body wall is still "moving" downward, covering the wound area (Fig. 6A). The body wall was now relaxed and the papulae protruded again, indicating that the wound was actually healed and possibly the internal hydrostatic pressure completely restored (Fig. 6A, B). The newly formed epidermis was much thicker and organized (Fig. 6C, D). The supporting cells, columnar in shape, were now more differentiated, bearing microvilli and cilia. Their roundish nucleus was basally positioned and their apical cytoplasm contained some dense or finely granular granules (Fig. 6D). Several secretory cells, filled by roughly circular electron-translucent granules (about $1.1 \mu \mathrm{m}$ in average diameter) were present. All 
around the bases of these cells, well differentiated neural processes could also be found: they were filled with a large number of small electron-dense vesicles (about $120 \mathrm{~nm}$ in diameter).

Just beneath the wound epidermis, clots of different cells, apparently formed by freemigrating elements, were intermixed with newly deposited collagen fibrils, leaving room for the formation of a wide empty area of oedematous area (Fig. 6E). The most numerous cell types were phagocytes, easily recognizable by their large size, irregular shape, and cytoplasmic content especially represented by large phagosomes (Fig. 6F). Furthermore widespread presumptive fibroblasts (Fig. 6G) and some scattered morula cells or spherule cells (type III, according to the classification by Chia and Xing (27)) were also found, these latter containing translucent granules in their cytoplasm. In addition to these cytotypes, several altered myocytes, released by the injured muscles, were found widespread in the oedematous area (Fig. 6H). These myocytes were undergoing evident dedifferentiation processes, often characterized by the presence of typical "spindle-like" structures (SLSs; Fig. 61) in which extensive rearrangement and packaging of their contractile apparatus were occurring. Some SLSs were ingested by phagocytes. In some cases only the contractile apparatus was ingested. Apparent flows of different cell types moved from the stump (upward from the RNC area and downward from the aboral wall) to the oedematous area. Free circulating cells were also visible in the perivisceral coelom and the papulae.

\section{Discussion}

In this work we described the basic cellular mechanisms occurring during the repair phase in the Mediterranean red starfish E. sepositus following traumatic arm amputation.

Within one hour p.a. an immediate emergency reaction occurred. The tip of the stump was strongly constricted to seal off the perivisceral coelomic canal from the outer environment, acting like a haemostatic (coelomo-static) ring: this prevented excessive loss of coelomic fluid 
and, at the same time, restored and maintained the internal physiological hydrostatic pressure in the rest of the body. This was evidenced by the different conditions shown by the papulae (which are expansions of the perivisceral coelom): in fact, the papulae nearest to the coelomostatic ring looked deflated, whereas the more distant ones maintained their functional turgidity. In contrast to the papulae, those tube feet nearest to the wound surface conserved their turgor, thus indicating that the sealing of the water vascular canal was more effective and rapid than that of the perivisceral coelom.

The emergency reaction was apparently achieved by a synergic activation of different components, namely coelomocytes, muscles and scattered dermal myocytes and, possibly, also the dermal MCT. The rapid coagulation of the coelomic fluid was achieved by coelomocytes: they rapidly formed a clot of cells, facilitating the closure of the injured perivisceral coelomic canal. Only the mature coelomocytes were able to aggregate using their filopodial processes (28). The coelomocytes coagulating in the canal were probably those already present in the coelomic liquid. However, this does not exclude the possibility that the CE produces coelomocytes immediately after wounding since the transition to the filopodial form arises within 5 to 8 minutes in fresh preparations and suspended cells (28).

Together with coelomocytes, also myocytes were actively involved in the emergency reaction in terms of both muscular activity and cell recruitment. In terms of muscle contraction, this was in agreement with previous work carried out in Leptasterias hexactis where the loss of coelomic fluid is similarly stopped also by a strong contraction of the arm stump (11). In our samples, this contraction was performed mainly by the circular muscle layer beneath the CE, which formed an internal haemostatic ring surrounding the coelomic cavity. Nevertheless, this event did not completely explain the overall constriction of the whole arm wall (i.e. including the aboral dermis and epidermis), its apparent reduction in thickness and the presence of a more compact dermis. This could be reasonably explained by an involvement of the myocyte 
network within the dermal layer: a myocyte contraction is likely to remodel the collagen bundles to which these are structurally connected. The function of the associated CE-derived granulocytes remains unknown, although their invariable co-existence with myocytes suggests a functional relationship. Although highly speculative, the occasional presence of presumptive juxtaligamental cells might suggest that the muscle contraction (both of the circular bundles and the dermal network) can be further supported by a stiffening of the mutable collagenous tissue (MCT) composing the dermis of the arm wall (22). Indeed, in many cases echinoderm MCTs can contribute or even substitute muscle functions as energy sparing mechanism during posture maintenance (e.g. in crinoids; 24). This might be particularly important in the initial regenerative phase of E. sepositus, since experimental starfish do not feed during the first weeks post amputation (personal observations).

Besides sealing off the coelom, the stump contraction reduced the injury surface, thus accelerating wound cicatrisation. This was helped also by a further downward folding of the aboral surface (which apparently slipped toward the oral side), that made the wound edges closer and increased protection of the injured site. Within $24 \mathrm{~h}$ p.a. the wound healing process began: this is initially achieved recruiting and recycling the adjacent epidermal cells which were stretched and migrated over the wound, creating a thin epithelium already provided with cell junctions. It is not clear whether this epithelial covering is driven by the underlying and developing nervous plexus or by the epidermal cells themselves. The wound closure was supported by the formation of a phagocyte syncytial network separating the developing epithelium from the injured stump tissues which started to exhibit an active rearrangement process. It is well known that the fusion of phagocytic cells usually occurs after the contact between the coelomic fluid and the external environment (29). In E. sepositus, the cell syncytia could be considered as the first barrier against loss of coelomic fluid following trauma. Also, it could represent a mechanism widely used by many organisms to eliminate 
foreign bodies; a syncytial-like structure has been found in the starfish Acanthaster planci following an injection of bile slats and other contaminants (30). To the best of our knowledge, such phagocyte network formation during wound healing in invertebrates has never been documented before. However, this phenomenon is reminiscent of the formation of a network of blood vessels, which is a critical component of wound healing in mammals. The endothelial cells migrate, invade the ECM stroma, and form tube-like structures that continue to extend, branch, and create networks, which supply oxygen and nutrients to cells that are rebuilding the affected tissue (31). By $72 \mathrm{~h}$ p.a., the wound epithelium was almost completely differentiated, thicker and permanent: epidermal cells acquired their final morphological and functional features (prismatic shape, microvilli and cilia on the outer surface, secretory granules in the cytoplasm) and an underlying nerve plexus was well developed. The wound covering occurred in a centripetal direction, towards the $\mathrm{RNC} /$ water vascular canal area. This behaviour has been already observed during the ophiuroid repair phase (15) and suggests that the injured $\mathrm{RNC}$ or water vascular canal area released chemo-attractant molecules that direct or attract the migration of wandering cells. It is well known that echinoderm regeneration is a nerve-dependent phenomenon (32).

At this time an oedematous area was formed behind the newly established epidermis, composed of several cytotypes which were characterized by transmission electron microscopy (TEM). Among all intermixed cells, phagocytes represented the most abundant population, as has been described in the common sea star A. rubens (28). Phagocytes are recruited at the beginning of the regeneration process and, although their number considerably varies within taxa, their involvement is usually related and restricted to the repair phase, in both arm and visceral echinoderm regeneration (33). The main characteristic of these cells is their ability to phagocytize other cells or foreign particles (34). The phagocytosis of foreign materials in $E$. sepositus initiated within 24 hours following amputation. Phagocytosis is an important feature 
of the immune response throughout the animal kingdom: in fact, it represents the first line of defence (35). It has been demonstrated that phagocytes in the sea star Asterias vulgaris can remove $2.6 \times 10^{7}$ sea urchin coelomocytes within one hour when sea urchin cells are injected into the body cavity of the sea star (36). Moreover, phagocytes can degrade not only foreign materials, but also their own particles (debris) or cells when necessary. In E. sepositus phagocytes began to digest SLSs/degenerated myocytes present in the oedematous area or in the area close to the wound site after $72 \mathrm{~h}$ p.a.: indeed, most of these degenerating myocytes came from injured muscles at the level of the amputation plane. The first stages of dedifferentiation included the tight packaging of myofilaments in defined sarcoplasmic areas which could be eventually removed by exocytosis and subsequently ingested by phagocytes. Similar means of recycling myocytes has been described in the holothurian Holothuria glaberrima following evisceration of the digestive tract (37).

In terms of cell recruitment for regeneration, several authors have shown that myocyte dedifferentiation is a common event in echinoderm regeneration processes and that the dedifferentiated cells might play an important role in the formation of the new tissues or organs (8). Indeed, in echinoderms myocytes are the most "plastic" cytotype to be used and recycled for a potential use during the regrowth phase and this remarkable ability might be one of the key aspects of their striking regenerative capacity. It is still not clear if myocyte dedifferentiation gives rise directly to new populations of cells or if they are involved in complicated processes mediated by phagocytes in which they undergo degeneration and mainly contribute indirectly as source of important cell reserves (e.g. proteins). Tracking the fate of "myocytes" after myofilament expulsion would certainly help to clarify this issue. Regardless the implicated mechanism, direct or indirect, it is noteworthy that these processes of rearrangement/dedifferentiation at the level of the muscles were closely associated with massive cell migration. 
The crucial role of phagocytes and their abundance in the oedematous area did not exclude the presence and the importance of other cell types, such as morula cells. According to Chia and Xing's (27) classification, we have identified a morula cell (spherule cell) type III whose granules seem to be void of content. Morula cells are reported to participate in the synthesis of a large number of humoral factors of the echinoderm immune system and inflammatory responses (38), as well as being associated with antibacterial activity (39), extracellular matrix remodeling (40) and wound healing (41). Other cytotypes could be present in the oedematous area, such as fibroblasts, since new extracellular matrix (ECM) including collagen was deposited progressively beneath the wound epithelium. As in mammalian wound healing processes (42), in E. sepositus the ECM started to be deposited after the "immune response" phase, but was delayed in comparison with them. The new collagen fibrils were widespread in the oedematous area in a still partly disorganized pattern, complete organization being reached in the following regenerative stages. In contrast to mammalian cases (43) in which fibrosis and/or over-deposition of collagen are detectable, in our samples from the wound area no signs of these phenomena were visible. As suggested for other echinoderm classes (e.g. Holothuroidea (44), the delayed deposition of collagen and other ECM components in comparison with mammalian events might explain the effectiveness of the regenerative processes in this Phylum.

The initial healing events occurred also at the level of the nerve: a thin layer of nervous tissue extending into the wound area of the regenerating arm-tip of E. sepositus was detectable within $24 \mathrm{~h}$ p.a. The migration of cells of the radial nerve stump to cover the wound site was accompanied by disorganization of the neurofibrillar zone of the RNC which remained over 7 days. At this stage the hyponeural system did not show any evident sign of regeneration. This delay could be related to its function, i.e. the innervation of the effector systems, such as tube feet, which will be regenerated later. It is well known that in many echinoderm regeneration 
models, the RNC starts to undergo repair first and subsequently drives the whole regenerative process (32).

In conclusion, in E. sepositus the repair phase lasted three days, during which initial wound healing occurred followed by an initial accumulation of a variety of cells, mainly phagocytes, forming an oedematous area just beneath the wound epidermis. Figure 7 summarizes the most important events of these early stages. The regenerative process could be classified as morphallactic due to the absence of a true undifferentiated and localized blastema and to the remarkable rearrangement phenomena at the level of stump tissues (mainly the injured muscles). However, on the basis of what could be inferred from histological studies, the origin of most cells involved in arm regeneration was still ambiguous. It could be that cells of mixed origin might be recruited from more distant sources of stem/progenitor cells as suggested in $A$. rubens arm regeneration (18). To help to elucidate these and other ambiguities further studies on this starfish species using markers for cell proliferation, cell ageing or cell identity (e.g. myocyte markers) are strongly recommended.

\section{Acknowledgements}

This research was founded by Young Researcher Grant (University of Milan, PI: Dr. Michela Sugni). We would like to deeply thank the CIMA Center (Centro Interdipartimentale Microscopia Avanzata, University of Milan) for technical support in electron microscopy analyses. We are grateful to the Marine Protected Area of Portofino (Ligurian Sea, Italy) for permission to collect experimental animals and to the scuba divers Dario Fassini and Livio Leggio for the collection.

\section{Conflict of interest disclosure}

The authors certify that there is no conflict of interest with any financial organization regarding the material discussed in the manuscript. 
List of abbreviations:

CE- Coelomic Epithelium

DCT- Dense Connective Tissue

ECM- Extracellular Matrix

h p.a.- hour(s) post-amputation

HMDS- Hexamethyldisilazane

JLCs- Juxtaligamental Cells

LCT- Loose Connective Tissue

MCT-Mutable Collagenous Tissue

RNC- Radial Nerve Cord

RWC- Radial Water Canal

SLSs- Spindle-Like Structures

SPAFG- Sucrose-picric acid-formaldehyde-glutaraldehyde

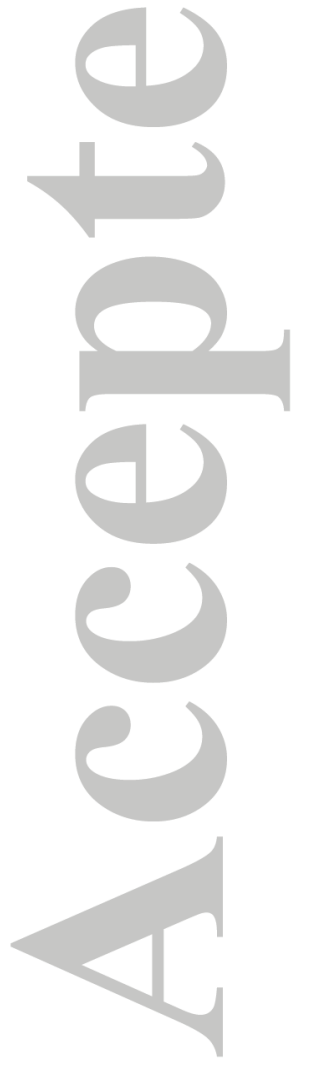

20

Wound Repair and Regeneration

This article is protected by copyright. All rights reserved. 


\section{References}

1. Goss RJ. Principles of regeneration. New York: Academic Press, 1969.

2. Mattson P. Regeneration. Indianapolis: Bobbs-Merrill, 1976.

3. Brockes JP and Kumar A. Comparative aspects of animal regeneration. Annu Rev Cell Biol 2008; 24: 525-49.

4. Toshinori H, Nobuhiko M, Yoko U, Mitsumasa Okamoto, Hisato Kondoha. FGF2 triggers iris-derived lens regeneration in newt eye. Mech Develop 2004; 121: 519-526.

5. Stocum DL. Regenerative biology and medicine. San Diego: Elsevier/Academic Press, 2006.

6. Han M, Yang X, Lee J, Allan CH, Muneoka K. Development and regeneration of the neonatal digit tip in mice. Dev Biol 2008; 315: 125-135.

7. Tanaka EM, Reddien PW. The cellular basis for animal regeneration. Dev Cell 2011; 21: $172-185$.

8. Candia Carnevali MD. Regeneration in echinoderms: repair, regrowth, cloning. ISJ 2006; 3: $64-76$

9. Candia Carnevali MD, Burighel P. Regeneration in Echinoderms and Ascidians. In: eLS. John Wiley \& Sons Ltd, Chichester 2010.

10. Sea Urchin Genome Sequencing Consortium. The genome of the sea urchin Strongylocentrotus purpuratus. Science 2006; 413: 941-952.

11. Mladenov PV, Bisgrove B, Aostra S, Burke RD. Mechanisms of arm-tip regeneration in the sea star Leptasterias hexactis. Roux Arch Dev Biol 1989; 198: 19-28.

12. Moss C, Hunter J, Thorndyke MC. Pattern of bromodeoxyuridine incorporation and neuropeptide immunoreactivity during arm regeneration in the starfish Asterias rubens. Phil Trans R Soc Lond. B 1998; 353: 421-436. 
13. Ducati CC, Candia Carnevali MD, Barker MF. Regenerative potential and fissiparity in the starfish Coscinasterias muricata. In: Heinzeller T, Nebelsick JH, editors. Echinoderms. Proceedings of the 11th International Echinoderm Conference, 2003 Oct 6-10; München: Taylor \& Francis Group, London 2004; 113-118.

14. Candia Carnevali MD, Bonasoro F. Microscopic overview of crinoid regeneration. Microsc. Res. Techniq 2001; 55: 403-426.

15. Biressi A, Ting Zou, Dupont S, Dahlberg C, Di Benedetto C, Bonasoro F et al. Woundhealing and arm regeneration in Ophioderma longicaudum and Amphiura filiformis (Ophiuroidea, Echinodermata): comparative morphogenesis and histogenesis. Zoomorphology 2010; 129: 1-19.

16. Sugni M, Mozzi D, Barbaglio A, Bonasoro F, Candia Carnevali MD. Endocrine disrupting compounds and echinoderms: new ecotoxicological sentinels for the marine ecosystem. Ecotoxicology 2007; 16(1): 95-108.

17. Candia Carnevali MD, Bonasoro F. Introduction to the biology of regeneration in echinoderms. Microsc Res Techniq 2001; 55: 365-368.

18. Hernroth B, Farahani F, Brunborg G, Dupont S, Dejmek A, Nilsson, Sköld H. Possibility of mixed progenitor cells in sea star arm regeneration. J Exp Zool (Mol Dev Evol) 2010; 6: $457-468$.

19. Holm K, Dupont S, Sköld H, Stenius A, Thorndyke M, Hernroth B. Induced cell proliferation in putative haematopoietic tissues of the sea star, Asterias rubens (L.). J Exp Biol 2008; 211: 2551-2558.

20. Hyman LH. The Invertebrates, vol IV. Echinodermata. New York: McGraw-Hill, 1955. 21. Ben Khadra Y, Said K, Thorndyke M, Martinez P. Homeobox Genes Expressed During Echinoderm Arm Regeneration. Biochem Genet 2014; 52: 166-180. 
22. Smith AB. Biomineralization in echinoderms. Carter JG, editor. Skeletal Biomineralization: Patterns, Processes and Evolutionary Trends. New York: Van Nostrand Reinhold, 1990: 413-443.

23. Motokawa T. Mechanical Mutability in Connective Tissue of Starfish Body Wall. Biol Bull 2011; 221: 280-289.

24. Chia FS, Koss R. Echinodermata. In: Harrison FW, Chia FS, editors. Microscopic Anatomy of Invertebrates. Wiley-Liss, 1994: 169-245.

25. Wilkie IC. Mutable collagenous tissue: Overview and perspectives. In: Matranga V, editor. Echinodermata. Progress in Molecular and Subcellular Biology. Marine Molecular Biotechnology. Berlin: Springer, 2005: 221-250.

26. Viehweg J, Naumann W, Olsson R. Secretory Radial Glia in the Ectoneural System of the Sea Star Asterias rubens (Echinodermata). Acta Zool 1998; 79: 119-131.

27. Chia FS and Xing J. Echinoderm Coelomocytes. Zool Stud 1996; 35(4): 231-254.

28. Pinsino A, Thorndyke MC, Matranga V. Coelomocytes and posttraumatic response in the common sea star Asterias rubens. Cell Stress Chaperon 2007; 12: 331-341.

29. Isaeva VV, Korenbaum ES. Defense function of coelomocytes and immunity of echinoderms. Sov Mar Biol 1990;15: 353-363.

30. Grand A, Pratchett M, Rivera-Posada J. The Immune Response of Acanthaster planci to Oxbile Injections and Antibiotic Treatment. J Mar Biol 2014. doi: org/10.1155/2014/769356.

31. Tonnesen MG, Feng X, Clark RAF. Angiogenesis in Wound Healing. Nature 2000; 5: 4046.

32. Thorndyke MC and Candia Carnevali MD. Regeneration neurohormones and growth factors in echinoderms. Canadian Journal of Zoology 2001; 79: 1171-1208. 
33. Candia Carnevali MD, Thorndyke MC, Matranga V. Regenerating echinoderms; a promise to understand stem cells potential. In: Rinkevich B, Matranga V, editors. Stem cells in marine organisms. Heidelberg: Springer, 2009: 165-186.

34. Endean R. The coelomocytes and coelomic fluids. In: Boolootian RA, editor. Physiology of Echinodermata. New York: Intersciences, 1966.

35. Greenberg SS. Immunity and survival. New York: Human Sciences Press, 1989: 19-34.

36. Reinisch CL, Bang FB. Cell recognition: reactions of the sea star (Asterias vulgaris) to the injection of amoebocytes of sea urchin (Arbacia punctulata). Cell Immunol 1971; 2: 496-503. 37. García-Arrarás JE, Dolmatov IY. Echinoderms; potential model systems for studies on muscle regeneration Curr Pharm Des 2010; 16(8): 942-955.

38. Pagliara P and Canicatti C. Isolation of cytolytic granules from sea urchin amoebocytes. Eur J Cell Biol 1993; 60: 179-184.

39. Haug T, Kjuul AK, Styrvold OB, Sandsdalen E, Olsen OM, Stensvag K. Antibacterial activity in Strongylocentrotus droebachiensis (Echinoidea), Cucumaria frondosa (Holothuroidea), and Asterias rubens (Asteroidea). J Invertebr Pathol 2002; 81: 94-102.

40. García-Arrarás JE, Schenk C, Rodrigues-Ramirez R, Torres II, Valentin G, Candelaria AG. Spherulocytes in the echinoderm Holothuria glaberrima and their involvement in intestinal regeneration. Dev Dyn 2006; 235: 3259-3267.

41. San Miguel-Ruiz JE, García-Arrarás JE. Common cellular events occur during wound healing and organ regeneration in the sea cucumber Holothuria glaberrima. BMC Dev Biol 2007; 7: 115 .

42. Diegelmann RF, Evans MC. Wound healing: an overview of acute, fibrotic and delayed healing. Front Bioscience 2004; 9: 283-289.

43. Rahban SR, Garner WL. Fibroproliferative scars. Clin Plast Surg 2003; 30: 77. 
44. Cabrera-Serrano A, Garcìa-Arraràs JE. RGD-containing peptides inhibit regeneration in the sea cucumber Holoturia glaberrima. Dev Dyn 2004; 231: 171-178.

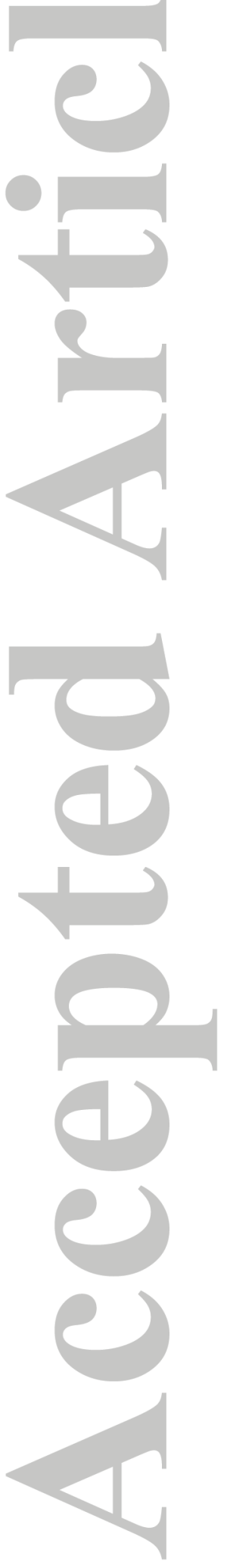




\section{Figure Legends}

Fig. 1. Main anatomical features of E. sepositus arm. A: A transverse section of E. sepositus arm-tip (light microscopy (LM)). B: SEM photo of an ossicle. C: TEM micrograph of the basal part of the coelomic myoepithelium (CE) consisting of peritoneocytes (arrow) and longitudinal myocytes and nerve processes (arrowheads). Abbreviations: cc-coelomic cavity, CE-coelothelium, o-ossicle, sp-spine, pl-papulae, gl-gland, tf-tube foot, ap-ampulla, RNCradial nerve cord, DCT-dense connective tissue, LCT-loose connective tissue, utam-upper transverse ambulacral muscle, lm-longitudinal myocyte. Scale bar (C): $1 \mu \mathrm{m}$.

Fig. 2. Ultrastructure of connective tissue cells. A: TEM micrograph of a typical connective tissue cell (arrow), and myoepithelial cells and granulocytes enclosed by a basal lamina (arrowhead). B: TEM micrograph of a myoepithelial cell (arrow), characterized by a prominent euchromatic nucleus and cytoplasm occupied by bundles of myofilaments, in strict association with a granulocyte (arrowhead) containing circular and homogeneously electrondense granules (asterisks). C: TEM micrograph of myoepithelial cells (arrow) occupied by bundles of myofilaments and granulocytes (arrowhead) with a massive cytoplasmic presence of roundish and homogeneously electron-dense granules (asterisks). D: Detail of cell processes between myoepithelial cells and granulocytes (chamber) where microvilli and cilia are observed. Abbreviations: c-collagen, f-myofilaments, ch-chamber, cl-cilia, n-nucleus. Scale bars (A-C): $1 \mu \mathrm{m}$.

Fig. 3. Main components of E. sepositus RNC. A: Ectoneural epithelium composition: a thin hyaline layer (arrowhead) covering the surface of the cells, a somatic zone (s.z) and a fibrillar zone (fb.z). B: TEM micrograph detailing the hyaline layer. C: Detail (TEM micrograph) of the basal part of the ectoneural epithelium showing the axial part of supporting cells which contain intermediate filament bundles, their final end-feet (arrowhead) and nervous processes (arrows) with small electron dense granules. Abbreviations: hy-hyaline layer, ecn-ectoneural 
epithelium, hn-hyponeural, sp-septum, s.z-somatic zone, fb.z-fibrillar zone, if-intermediate filaments. Scale bars: $1 \mu \mathrm{m}(\mathrm{B}), 2 \mu \mathrm{m}(\mathrm{C})$.

Fig. 4. Wound sealing (1 hour p.a.). A (stereomicroscopy (SM) view) and B (LM): haemostatic ring (arrow); the distal portion of the stump (about $3 \mathrm{~mm}$ ) strongly constricted. C: Normal unconstricted body wall. D: At the constriction the collagen fibres (asterisks) of the DCT become more packed, the coelothelium shows a folded profile (arrow) and the circular muscle fibres (arrowhead) appear contracted in comparison to norm (arrowhead) (C), E: Coelomocytes clotting in the coelomic cavity (arrows). F: TEM micrograph of a presumptive undifferentiated cell found in the coelomic cavity. G: Injured muscle showing first signs of reorganization and release of cells towards the wound area (arrow). Abbreviations: cccoelomic cavity, CT-connective tissue, lam-longitudinal ambulacral muscle, m-muscle, nnucleus, o-ossicle, pc-pyloric caeca, RNC-radial nerve cord, tf-tube foot. Scale bar (F): $1 \mu \mathrm{m}$.

Fig. 5. Wound healing (24 hours p.a.). A (SM view): The strong body wall contraction still persists (arrow). B (SM view): The aboral body wall converges and folds towards the oral side (arrow). C: Complete wound healing and the formation of a new thin epithelium (arrow). D (LM): Wound epidermis (arrowhead) and healing neuroepithelium (arrow). E: TEM micrograph of the new epidermis which is composed by a monolayer of dedifferentiated epidermal cells (arrow). On its inner epithelial side numerous presumptive nervous processes were found (arrowheads). F: Detail of Fig. E; Typical cell junction complexes (arrowhead) and small microvilli (arrow) of the new epithelium are present together with electron-dense granules (asterisks). G: Loose syncytial network of phagocytes beneath this wound epidermis. Abbreviations and symbols: c-collagen fibrils, cc-coelomic cavity, DCT-dense connective tissue, ECM-extracellular matrix, EX-external environment, gl-gland, lam-longitudinal ambulacral muscle, lac-lacunar area, LCT-loose connective tissue, n-nucleus, o-ossicle, pc- 
pyloric caeca, pl-papulae, RNC-radial nerve cord, RWC-radial water canal, tf-tube foot, vvacuole, asterisk-electron-dense granule. Scale bars: $1 \mu \mathrm{m}(\mathrm{E}), 1 \mu \mathrm{m}(\mathrm{F}), 10 \mu \mathrm{m}(\mathrm{G})$.

Fig. 6. Oedematous area formation (72 hours p.a.). A (SEM micrograph): The aboral body wall movement downward (arrow). B (LM): The body wall is relaxed. C (SEM micrograph): The newly formed epidermis is much thicker and organized (arrow). D (TEM micrograph): The supporting cells are more differentiated (bearing microvilli and cilia (arrow)) and their apical cytoplasm contains some dense or finely granular granules (arrowhead). The secretory cells filled by electron-translucent granules (asterisks). E (LM): Oedematous area: Intermixed cells beneath the wound epithelium (arrows). F: TEM micrograph of a phagocyte. G: TEM micrograph of a presumptive fibroblast with GA in full activity and an evident nucleolus. H: TEM micrograph of dedifferentiated myocytes found in the oedematous area (arrows). I: Detail of "Spindle-like" structures (SLSs) of a myocyte. Abbreviations: c-collagen, cccoelomic cavity, CT-connective tissue, DCT-dense connective tissue, GA-Golgi apparatus, glgland, oe-oedematous area, lam-longitudinal ambulacral muscle, nCT-newly deposited collagen, ne-new epidermis, o-ossicle, oe-oedematous area, pc-pyloric caeca, pl-papulae, RNC-radial nerve cord, tf-tube foot. Scale bars: $10 \mu \mathrm{m}(\mathrm{D}), 2 \mu \mathrm{m}(\mathrm{F}, \mathrm{I}), 1 \mu \mathrm{m}(\mathrm{G}), 5 \mu \mathrm{m}(\mathrm{H})$.

Fig. 7. Diagram summarizing the main events during E. sepositus repair phase. A: gross morphology of non-regenerating arm. B: Wound sealing (1 h p.a.): a haemostatic ring formed by circular muscle and aboral collagen fibre contraction, and coelomocytes clotting in the coelomic cavity. C: Wound healing (24 h p.a): Re-epithelialisation, D: Oedematous area formation (72 h p.a.): pool of various cells (myocytes, phagocytes...) intermixed with newly deposited collagen fibrils. 